\title{
Comité consultatif national de l'immunisation (CCNI) : Célébration de 50 ans de service
}

\author{
Gemmill I. ${ }^{*}$ \\ ${ }^{1}$ Président du CCNI, Kingston (Ontario) \\ *Auteur-ressource : naci-ccni@phac-aspc.gc.ca
}

\section{Résumé}

Le Comité consultatif national de l'immunisation (CCNI) célèbre son cinquantième anniversaire en octobre 2014. Le présent document décrit l'historique du CCNI et ses activités au cours des 50 dernières années. Après sa formation en 1964, le CCNI a entrepris l'élaboration de lignes directrices pour les quelques vaccins qui étaient approuvés à l'époque, y compris les vaccins contre la poliomyélite, la rougeole, le tétanos, la coqueluche, la diphtérie et la variole. Même si le Comité a évolué au fil des ans, il a continué à mettre l'accent sur l'offre d'orientation sur un nombre toujours croissant de produits : vingt-quatre, selon les récentes mises à jour apportées au Guide canadien d'immunisation.

Avec l'évolution du contexte de vaccination, y compris les pratiques et les programmes d'immunisation, la composition des membres du Comité a évolué pour inclure une expertise plus approfondie, comme l'immunologie. Le Comité a dû adapter son travail, en raison de données probantes plus complexes et abondantes et de l'établissement d'une méthodologie fondée sur les données probantes publiées. Au cours des 50 dernières années, la réussite et les accomplissements du CCNI ont été en grande partie attribuables aux efforts de ses présidents et de ses membres, qui ont consacré des heures de travail infinies aux activités et aux produits du Comité. Les groupes consultatifs techniques du CCNI sont reconnus à l'échelle mondiale comme une ressource précieuse qui est bénéfique pour les pratiques et les programmes liés à l'immunisation partout au Canada et à l'échelle internationale. Le CCNI est un comité dont l'ASPC et, en fait, le Canada peuvent être réellement fiers.

\section{Introduction}

Le Comité consultatif national de l'immunisation (CCNI) célébrera son cinquantième anniversaire en octobre 2014. Le présent document expose la formation, l'historique et le travail du CCNI au cours des 50 dernières années. Le Comité a été formé en 1964 en tant que Comité consultatif national sur les agents immunisants par le ministère national de la Santé et du Bien-être social d'alors, et il dépendait du Conseil fédéral, provincial, territorial d'hygiène. Au cours des 50 dernières années, le CCNI a fourni des conseils fondés sur des données probantes sur les vaccins dont l'utilisation est autorisée au Canada, ainsi que sur les pratiques liées à l'immunisation et les calendriers de vaccination, à l'aide d'examens méticuleux et minutieux et du meilleur jugement de certains des professionnels les plus compétents en pédiatrie, en soins infirmiers, en maladies infectieuses, en immunologie et en santé publique au Canada. Le CCNI a été un modèle pour d'autres comités consultatifs d'experts et comités consultatifs sur les politiques au Canada et il est réputé comme l'autorité en matière de pratique de l'immunisation dans ce pays.

\section{Débuts}

Le CCNI a tenu sa première réunion en octobre 1964 et il était présidé par le $D^{r}$ Andrew Rhodes, virologiste canadien hautement respecté de l'Université de Toronto. Un groupe technique avait été réuni afin de discuter de la mise en œuvre du vaccin antipoliomyélitique au Canada plusieurs années plus tôt, et le $D^{r}$ Rhodes croyait qu'il était nécessaire qu'un groupe fournisse des conseils de façon continue sur les vaccins contre la poliomyélite et les autres vaccins de l'époque, comme le vaccin contre la rougeole. En avril 1963, une présentation a été faite au Conseil fédéral d'hygiène (groupe de sous-ministres qui était à la tête des programmes de santé au Canada), qui 
a approuvé la constitution d'un comité « en qualité de Comité consultatif sur les vaccins ». En 1977, le nom a été modifié pour Comité consultatif national de l'immunisation (CCNI), qui reflète le fait que le Comité formule des recommandations sur les vaccins, ainsi que sur les pratiques et les programmes liés à l'immunisation.

« L'objectif du Comité se présentait [présente] en deux volets : premièrement, donner des avis et formuler des recommandations sur les agents immunisants au Ministre par l'intermédiaire du Conseil fédéral d'hygiène, à la demande du Conseil ou de son président, et deuxièmement, proposer au Conseil des aspects des agents immunisants qui semblent nécessiter une attention particulière... » Bien que le cadre de référence du CCNI a été mis à jour à de nombreuses occasions au fil des ans, ces deux objectifs reflètent toujours l'esprit du travail fondamental du Comité.

Le $D^{r}$ Rhodes possédait manifestement une vision perspicace. En 1964, il n'existait qu'une poignée de vaccins. Le vaccin contre la variole était utilisé depuis des décennies, le vaccin contre la coqueluche depuis 1918, le vaccin contre la diphtérie depuis les années 1920 et le vaccin contre le tétanos depuis les années 1940 (1). Les vaccins contre la poliomyélite et la rougeole étaient nouveaux. Aujourd'hui, la version en ligne du Guide canadien d'immunisation (2) fournit de l'orientation sur 24 vaccins et on en prévoit beaucoup plus. En outre, de temps à autre, le CCNI fournit des conseils sur la mise en place de programmes universels, comme le vaccin contre l'hépatite B pour les enfants et il a fourni des conseils sur les meilleures pratiques en matière d'immunisation, de la façon de faire une injection intramusculaire (IM) adéquate, à la communication efficace avec les sujets vaccinés. De plus, on pourrait ultérieurement demander au CCNI de fournir de l'orientation sur d'autres éléments comme le rapport coût-efficacité de différents programmes de vaccination. La portée des travaux du CCNI s'est grandement élargie au cours des 50 dernières années et il est difficile d'imaginer comment les professionnels de la santé et les personnes responsables de la mise en œuvre de programmes de vaccination s'en sortiraient sans

ses conseils. Le système de santé est chanceux de posséder un éminent organisme comme le CCNI pour fournir des conseils détaillés et objectifs sur l'immunisation.

\section{Membres et présidence du CCNI}

La composition des membres du CCNI a évolué au fil des ans. Les premiers membres étaient des grands noms en virologie, bactériologie et pédiatrie, ainsi que des administrateurs de santé publique fédéraux et provinciaux. En 1980, un professionnel de la santé publique local a été ajouté. Des experts en maladies infectieuses ont été invités à se joindre au Comité et un immunologiste a été recommandé dès 1976 (le CCNI compte maintenant un immunologiste comme membre permanent). Depuis les années 1970, diverses organisations professionnelles œuvrant dans la pratique de l'immunisation ont été invitées à nommer des représentants de liaison, qui non seulement fournissent un lien à ces autres organisations, mais qui apportent aussi des connaissances approfondies et une grande expérience lors des délibérations du CCNI. Quelques exemples de ces organisations professionnelles sont énumérés dans le Tableau 1. 
Tableau 1 : Organisations de liaison représentées au sein du CCNI au cours des dernières décennies

- Comité consultatif de l'épidémiologie

- Advisory Committee on Immunization Practices, Centers for Disease Control and Prevention, Atlanta

- Association pour la microbiologie médicale et l'infectiologie Canada

- Association canadienne pour la recherche et l'évaluation en immunisation

- Comité canadien sur l'immunisation

- Société canadienne des maladies infectieuses

- Association médicale canadienne

- Coalition canadienne des infirmières et infirmiers pour l'immunisation

- Société canadienne de pédiatrie

- Association canadienne de santé publique

- Association canadienne des infirmières et infirmiers en santé du travail

- Collège des médecins de famille du Canada

- Association pour la prévention des infections à l'hôpital et dans la communauté

- Conseil des médecins hygiénistes en chef

- Comité consultatif de la médecine tropicale et de la médecine des voyages

- Société des obstétriciens et gynécologues du Canada

Le CCNI a été mené par une série d'éminents professionnels de la santé qui ont servi de président et qui ont conféré une direction visionnaire aux travaux et aux discussions du CCNI (Tableau 2). Malheureusement, le président qui a occupé son poste le plus longtemps, $D^{r}$ Michael Dixon, est décédé en novembre 2013, moins d'un an avant le cinquantième anniversaire du CCNI. Dr Dixon a occupé le poste de président pendant 17 ans, de 1972 à 1989.

Tableau 2 : Présidents du Comité consultatif national de l'immunisation

\begin{tabular}{|c|c|}
\hline $1964-1966$ & $\mathrm{D}^{\mathrm{r}}$ Andrew Rhodes, Toronto (Ontario) \\
\hline $1968-1969$ & $D^{r}$ Edward Bynoe, Ottawa (Ontario) (par intérim) \\
\hline $1972-1989$ & $D^{r}$ J. Michael S. Dixon, Edmonton (Alberta) \\
\hline $1989-1993$ & $\mathrm{D}^{\text {re }}$ Susan Tamblyn, Stratford (Colombie-Britannique) \\
\hline $1993-1998$ & $D^{r}$ David Scheifele, Vancouver (Colombie-Britannique) \\
\hline $1998-2003$ & $D^{r}$ Victor Marchessault, Ottawa (Ontario) (décédé le 31 mars 2003) \\
\hline $2003-2007$ & $\mathrm{D}^{\text {re }}$ Monika Naus, Vancouver (Colombie-Britannique) \\
\hline $2008-2011$ & $D^{\text {re }}$ Joanne Langley, Halifax (Nouvelle-Écosse) \\
\hline $2011-2014$ & $\mathrm{D}^{\mathrm{re}}$ Bryna Warshawsky, London (Ontario) \\
\hline $2014-$ & $D^{r}$ lan Gemmill, Kingston (Ontario) \\
\hline
\end{tabular}




\section{Travaux du CCNI}

Les travaux réalisés par le CCNI étaient principalement axés sur la publication de lignes directrices sur les vaccins et les pratiques d'immunisation au moyen de déclarations sur les nouveaux vaccins ou nouvelles directives sur les vaccins établis et de la principale référence du CCNI pour l'utilisation des vaccins au Canada, le Guide canadien d'immunisation. Les déclarations sur les vaccins sont nécessaires lorsque l'utilisation d'un nouveau vaccin est autorisée dans le but d'appuyer la prise de décision avec des conseils crédibles, fondés sur des données probantes. Les déclarations du CCNI ont comblé ce besoin depuis la création du Comité en 1964. Les déclarations sont élaborées d'abord par un examen exhaustif du document, puis par la rédaction de lignes directrices fondées sur les meilleures données probantes, dans un format standard (3).

Anciennement, les déclarations étaient rédigées par des membres individuels, mais plus récemment, comme la complexité des produits d'immunisation a augmenté et qu'on a observé la prolifération de produits semblables afin de protéger la population contre les mêmes maladies, des groupes de travail ont été établis pour créer un plus large mécanisme pour accomplir cette tâche complexe. Il est important de comprendre que les recommandations du CCNI ne sont pas fondées simplement sur les monographies de produit, mais sur toutes les données probantes disponibles sur un vaccin donné. Étant donné la complexité accrue des produits d'immunisation, on observe également une augmentation similaire du volume et de la complexité des données cliniques et épidémiologiques que le CCNI évalue et classifie afin de formuler ses recommandations. Les déclarations sont rédigées avec soin et font l'objet d'un débat en profondeur. De plus, les lecteurs des déclarations tirent avantage du bon jugement des membres, qui tiennent compte de la faisabilité théorique et pratique et qui se concentrent précisément sur ce qui constitue la meilleure pratique pour les sujets vaccinés. Une fois que l'auteur principal et le groupe de travail se sont entendus sur les recommandations les plus raisonnables fondées sur des données probantes, la déclaration fait l'objet d'une discussion par tous les membres du CCNI aux fins d'approbation finale. Au fil des ans, des déclarations ont été formulées sur les nouveaux vaccins, sur les avancées dans la documentation sur divers vaccins et sur les questions liées à la vaccination, notamment le thimérosal.

Le Guide canadien d'immunisation a été publié pour la première fois en 1979, sous le nom Guide de vaccination à l'intention des Canadiens, et a été révisé tous les quatre ans en moyenne. Le guide fournissait des " commentaires et des conseils sur l'utilisation de tous les agents d'immunisation homologués pour les maladies infectieuses qui sont offerts au Canada au moment de la rédaction » (4). Le Comité pensait « que cette brochure contribuerait [contribuera] à l'atteinte d'un plus grand degré d'uniformité des politiques et des pratiques d'immunisation au Canada que celui atteint par le passé ». La version anglaise de la première édition comptait 92 pages, tandis que la version française comptait 104 pages. La septième édition, publiée en 2006, comptait près de 400 pages et offrait des conseils non seulement sur des vaccins précis, mais également sur les questions allant de l'utilisation de divers vaccins au sein de populations particulières, à l'innocuité des vaccins. Étant donné la complexité croissante de la pratique de l'immunisation et le taux rapide du changement dans les connaissances ainsi que les progrès réalisés au chapitre de la technologie qui ont facilité la production d'une version électronique du Guide canadien d'immunisation, le CCNI a entrepris la création d'une édition en ligne, qui peut facilement être modifiée et mise et à jour. Le travail sur l'édition en ligne a été effectué au début de 2014 et on prépare actuellement les calendriers pour les changements ponctuels, provoqués par la modification des connaissances, et les examens systématiques sur un cycle de quatre ans. 


\section{Conclusion}

Le CCNI a été une véritable institution en vaccinologie au Canada pendant la moitié d'un siècle. Il a fourni des conseils fiables et avant-gardistes qui dépassent les exigences juridiques d'une monographie de produit. II a survécu au Conseil qui l'a créé. Il a été une ressource pour l'Agence de la santé publique du Canada (ASPC) et ses prédécesseurs pendant une plus longue période que la plupart des comités et il continue de prospérer. Les professionnels de la santé qui ont participé au CCNI ont fourni des heures de travail infinies pour s'assurer que les professionnels de la santé canadiens possèdent les meilleurs conseils sur les pratiques d'immunisation, et ils considèrent comme un honneur leur nomination à cet éminent comité. En fait, il est considéré comme l'un des comités les plus importants du système de soins de santé au Canada, et il a établi la norme pour fournir des lignes directrices fondées sur des données probantes.

La nécessité et le rôle de ces comités consultatifs techniques sont reconnus à l'échelle mondiale. Sans l'orientation du CCNI au fil des ans, le système d'immunisation au Canada serait probablement plus chaotique et beaucoup moins uniformisé qu'il ne l'est aujourd'hui. L'Organisation mondiale de la Santé recommande l'établissement, le soutien et le renforcement de comités consultatifs nationaux sur l'immunisation en tant qu'élément clé à l'amélioration des programmes d'immunisation, à l'établissement de priorités ainsi qu'à l'introduction de nouveaux vaccins et de technologies de vaccination (5). En ce cinquantième anniversaire du $\mathrm{CCNI}$, c'est le moment de célébrer ses contributions au cours de la dernière moitié de siècle et de remercier les nombreux professionnels de la santé qui ont donné de leur temps et une attention réfléchie aux questions liées à l'immunisation au nom de nous tous ainsi que toutes les personnes engagées de l'Agence de la santé publique et de ses prédécesseurs qui ont appuyé le CCNI. Le CCNI est un comité dont l'ASPC et, en fait, le Canada peuvent être réellement fiers.

\section{Remerciements}

Les auteurs tiennent à remercier le travail réalisé précédemment par le $D^{r}$ Andrew Sherrington sur l'historique du $\mathrm{CCNI}$, qui a été largement utilisé pour rédiger ce commentaire.

\section{Références}

(1) Association canadienne de santé publique. Chronologie de l'immunisation. Accès : http://www.cpha.ca/fr/programs/history/achievements/12-v/timeline.aspx

(2) Agence de la santé publique du Canada. Guide canadien d'immunisation. Ottawa : Agence de la santé publique du Canada. Accès : http://www.phac-aspc.gc.ca/publicat/cig-gci/index-fra.php

(3) Comité consultatif national de l'immunisation. Recommandations pour l'immunisation fondées sur des données probantes - Méthodes du Comité consultatif national de l'immunisation. Relevé des maladies transmissibles au Canada. Janvier 2009; vol. 35(DCC-1).

(4) Santé et Bien-être social Canada. Guide canadien d'immunication. Ottawa: Approvisionnements et Services Canada; 1980.

(5) National Immunization Technical Advisory Groups (NITAGs): Guidance for their establishment and strengthening. Duclos P. Vaccine. 2010; 28(Supplement 1); A18-25. 